\title{
RELAÇÕES ENTRE SATISFAÇÃO COM A VIDA, COM A FAMÍLIA E COM AS AMIZADES E RELIGIOSIDADE NA ADOLESCÊNCIA
}

\author{
Doralúcia Gil da Silva \\ Universidade Federal do Rio Grande do Sul \\ Jaqueline Portella Giordani \\ Universidade Federal do Rio Grande do Sul \\ Débora Dalbosco Dell'Aglio \\ Universidade Federal do Rio Grande do Sul
}

\begin{abstract}
Resumo
Este trabalho teve por objetivo discutir relações entre satisfação de vida, satisfação com a família e com as amizades e religiosidade em amostra de adolescentes. Foram aplicadas: uma ficha de dados sociodemográficos, a Escala Multidimensional de Satisfação de Vida e a Escala de Afetos Positivos e Negativos para Adolescentes em 420 estudantes (média de idade $=14,91 ; \mathrm{DP}=1,65$ ) de escolas públicas de Porto Alegre. Os resultados indicaram que adolescentes que relataram ter religião apresentaram maior satisfação de vida, bem-estar subjetivo, satisfação com a família e com as amizades, com diferenças significativas. Religiosidade e satisfação com os relacionamentos interpessoais são fatores que podem atuar de forma positiva e incrementar a percepção de satisfação de vida pelo adolescente. Implicações na forma de avaliar o bem-estar subjetivo, assim como a religiosidade, são discutidas.
\end{abstract}

Palavras-chave: bem-estar subjetivo; adolescência; religiosidade.

\section{RELATIONS BETWEEN LIFE SATISFACTION, FAMILY AND FRIENDSHIP SATISFACTION AND RELIGIOSITY IN ADOLESCENCE}

\begin{abstract}
This work aimed to discuss relations between life satisfaction, family and friendship satisfaction and religiosity in a sample of adolescents. A record of sociodemographic data, a Multidimensional Scale of Life Satisfaction and a Positive and Negative Affect Scale were applied in 420 students $(M=14.91$ years old; $S D=1.65)$ from public schools of Porto Alegre. The results indicated that adolescents who reported having religion had higher life satisfaction, subjective well-being, and family and friendship satisfaction, all with significant differences. Religiosity and interpersonal relationships are factors that can act in a positive way and increase well-being perception by the adolescent. Implications about the form of to assess subjective well-being, as well as religiosity, are discussed.
\end{abstract}

Key words: subjective well-being; adolescence; interpersonal relationships. 


\title{
RELACIONES ENTRE SATISFACIÓN CON LA VIDA, CON LA FAMILIA Y LAS AMISTADES Y RELIGIOSIDAD EN LA ADOLESCENCIA
}

\begin{abstract}
Resumen
Este trabajo tuvo por objectivo discutir relaciones entre satisfacción de vida, con la familia y con las amistades y religiosidad en una muestra de adolescentes. Fueran utilizadas una ficha de datos sociodemográficos, la Escala Multidimensional de Satisfação de Vida y la Escala de Afetos Positivos e Negativos para Adolescentes en 420 estudiantes (média de edad $=14,91$; $D P=1,65$ ) de escuelas públicas de Porto Alegre. Los adolescentes que reportaran tener religión tuvieron más satisfación de vida, bienestar subjetivo, satisfacción con la familia y con las amistades con diferencias significativas. Religiosidad y satisfación con relaciones interpersonales son factores que pueden atuar de forma positiva y aumentar la percepción de BES pelo adolescente. Implicaciones en la forma de evaluar el bienestar subjetivo son discutidas.
\end{abstract}

Palavras-clave: bienestar; adolescencia; relaciones interpersonales.

\section{INTRODUÇÃO}

A psicologia positiva considera aspectos de saúde das pessoas, de forma a promover e desenvolver condições e capacidades positivas do desenvolvimento saudável (Passareli \& Silva, 2007). Entre essas capacidades positivas está o bem-estar, o qual possibilita que as pessoas vivenciem suas vidas de forma prazerosa, com uma melhor percepção de si e dos outros e desfrutem de situações cotidianas e no relacionamento com pares (Diener, 1984).

Diener e Scollon (2014) defendem diferentes motivos para estudar o bemestar. Entre eles está o fato de que, segundo um levantamento realizado em 47 países com estudantes universitários, a maioria das pessoas elenca a felicidade como a coisa mais importante para si. Apesar de o bem-estar ter sido alvo de reflexões ao longo dos séculos, apenas na última metade do século XX foram iniciados estudos empíricos sobre o tema (Diener, 2000). Assim, atualmente as pesquisas fornecem indicadores a respeito do construto e da promoção de bemestar baseados em evidências científicas (De Neve, Diener, Tay, \& Xuereb, 2013).

As concepções teóricas dos estudos sobre bem-estar organizam-se na perspectiva do bem-estar eudemônico (bem-estar psicológico) e hedônico (bemestar subjetivo - BES). Ryan e Deci (2001) pontuam que a primeira perspectiva propõe que o bem-estar é o pleno funcionamento das potencialidades de uma pessoa, incluindo sua capacidade de pensar e de bom senso. Já a segunda propõe o bem-estar como felicidade, sendo esse avaliado positivamente quando alguém relata altos níveis de satisfação com sua vida, altos níveis de emoções positivas e baixos níveis de emoções negativas. No presente estudo, foi utilizada a perspectiva hedônica e o conceito de bem-estar subjetivo (BES). Contudo, destaca-se que parte dos estudos revisados sobre bem-estar realizados com adolescentes utiliza a outra perspectiva e o conceito do fenômeno.

Diener (1984) define o BES como uma avaliação cognitiva e emocional a respeito da própria vida feita por uma pessoa. O componente cognitivo 
corresponde ao conceito de satisfação de vida (SV). A avaliação pode ser feita sobre a vida de forma global, ou sobre os diferentes domínios dela (como família, trabalho, escola). Diener (1984) considera mais adequado avaliar a satisfação de vida a partir dos parâmetros selecionados pelos próprios indivíduos como importantes para afetar o seu bem-estar. Segundo o autor, o fator afetivo abrange os afetos positivos (AP) e negativos (AN). O AP é o quanto um indivíduo se sente ativo e entusiasmado, enquanto o AN corresponde ao quanto uma pessoa sente estados de humor aversivos como culpa ou raiva (Watson, Clark, \& Tellegen, 1988).

Desse modo, o BES insere-se na área de prevenção e promoção em saúde (Diener, 2000), o que justifica seu estudo e promoção em períodos iniciais da vida, principalmente em uma das etapas cruciais para o desenvolvimento social como a adolescência. As pesquisas internacionais e brasileiras têm identificado variáveis associadas ao bem-estar. Sexo e idade mostram ter um papel importante para o fenômeno (Coenders, Casas, Figuer, \& González, 2005; Reina, Oliva, \& Parra, 2010; Sarriera et al., 2012; Segabinazi et al., 2012). No entanto, outros aspectos, como a religiosidade e a satisfação com os relacionamentos interpessoais, também desempenham influência na avaliação do BES pelos jovens.

Estudo realizado com 5316 adolescentes de 12 a 16 anos da Espanha, Brasil e Chile (Casas et al., 2015), indica que a religiosidade deve ser considerada na avaliação do bem-estar, conforme as características culturais do local onde foi feita a coleta de dados. Ao mesmo tempo, para outros pesquisadores, a religiosidade contribui tanto para O BES que poderia se configurar como uma nova dimensão da satisfação de vida (Wills, 2009). De fato, há evidências de que BES e religiosidade estão correlacionados, sendo essa última considerada como um saliente componente do primeiro entre adolescentes (Abdel-Khalek, 2010). Em pesquisa realizada com 196 adolescentes de 14 a 16 anos na Turquia, os resultados mostraram relação significativa entre satisfação de vida, afetos positivos e participação em atividades religiosas, não tendo sido encontrada relação significativa dos afetos negativos com a prática religiosa (Eryilmaz, 2015). Esse dado vai ao encontro do achado de que pessoas religiosas têm registrado, em média, índices maiores de BES em relação a pessoas que se declaram sem religião (Silva \& Santos, 2013). No caso dos adolescentes, estudo realizado com 1.562 estudantes do Rio Grande do Sul encontrou que maior frequência de prática religiosa estaria associada a um maior bem-estar entre jovens (Souza et al., 2012). A hipótese é de que a religiosidade possa agregar respeito e suporte social, além de propósito e significado para a vida, aspectos que podem contribuir para níveis mais altos na avaliação do BES (Silva \& Santos, 2013). No entanto, tais estudos não deixam claro de que forma a religiosidade foi avaliada, apenas fazem menção aos jovens responderem se possuem afiliação ou prática religiosa ou não. 
Algumas pesquisas têm encontrado também associação entre satisfação com a qualidade do relacionamento com a família e religiosidade em adolescentes. Os resultados destes estudos têm indicado que o envolvimento religioso familiar pode tornar os laços afetivos mais estreitos e minimizar os conflitos e desentendimentos intrafamiliares (Becker, Maestri, \& Bobato, 2015; Rabinovich, Costa, Lins, \& Franco, 2008). A religiosidade ocuparia, então, papel importante na estruturação e organização familiar, sendo também fator protetivo para comportamentos de risco na adolescência (Van der Meer Sanchez, de Oliveira, \& Nappo, 2004).

As pesquisas vêm demonstrando outro componente que cumpre papel relevante na avaliação do BES em adolescentes, como a satisfação com os relacionamentos interpessoais, seja na família ou com o grupo de pares. Em estudo realizado com estudantes portugueses foi avaliado BES associado à comunicação com amigos e com os pais. Os adolescentes com médias mais elevadas de BES foram os que obtiveram também índices melhores de comunicação tanto com amigos quanto com os pais, indicando que os jovens mais felizes mantêm relações mais positivas não somente com os pares, mas também com a família (Tomé, Camacho, Matos, \& Diniz, 2011). Em estudo com 4877 jovens portugueses, foi observado que uma boa comunicação com os pais tem um maior efeito protetor sobre os comportamentos de risco do que uma boa comunicação com os amigos, e que adolescentes com boa comunicação com os pais e amigos revelaram serem mais felizes e satisfeitos com a vida (Tomé, Camacho, Matos, \& Simões, 2015).

Ainda a respeito do BES e satisfação com relacionamentos interpessoais, em estudo realizado na China foi verificada uma preferência por relacionamentos com familiares mais próximos aos mais distantes, no que se refere à avaliação do BES (Cheng, Li, Leung, \& Chan, 2011). Ademais, a valorização do relacionamento com o grupo de pares tem se mostrado um preditor de bemestar na adolescência, não havendo diferenças nesse aspecto entre sexos e idades (Sarriera et al., 2013; Serafini \& Bandeira, 2011). Entretanto, por vezes a satisfação com as amizades recebe maior importância por algum dos sexos, tal como no estudo de Huebner, Drane e Valois (2000), com 5545 estudantes de Ensino Médio estadunidenses, em que as meninas se disseram mais satisfeitas com as amizades do que os meninos. A pesquisa de Souza e Duarte (2013) realizada com 116 adolescentes no centro-oeste brasileiro, também mostrou maior satisfação das meninas com as amizades e apontou que a satisfação com as amizades, com a família e com relacionamentos amorosos em conjunto está mais relacionada com BES do que uma dessas dimensões isoladamente.

Tendo em vista tais considerações, observa-se a importância de discutir as relações entre a religiosidade e dimensões específicas da satisfação de vida, como a satisfação com a família e as amizades, que por sua vez compõem o bem-estar subjetivo. Dessa forma, este estudo teve por objetivo discutir as 
relações entre satisfação de vida, satisfação com a família, com as amizades e religiosidade em amostra de jovens de escolas públicas de Porto Alegre.

\section{MÉTODO}

Este estudo caracteriza-se ser um levantamento quantitativo e transversal.

\section{Participantes}

Participaram deste estudo 420 adolescentes com idades entre 12 e 18 anos $(M=14,91 ; D P=1,65)$, de ambos os sexos (sendo $62 \%$ do sexo feminino). Os participantes estudavam desde o sexto ano do Ensino Fundamental ao terceiro ano do Ensino Médio de escolas da rede pública de Porto Alegre/ RS, sendo que uma parte deles $(9,9 \%)$ também relatou que trabalha. A maioria era solteira $(98,8 \%)$ e apenas $1,1 \%$ referiram viver com companheiro. Em relação à religião, os participantes respondiam se tinham alguma religião ou não e, em caso afirmativo, identificavam qual era. Sendo assim, 31,7\% dos adolescentes se declararam católicos, 12,4\% evangélicos, 5,9\% espíritas, 5,4\% umbandistas e $30,3 \%$ declararam não ter nenhuma religião.

\section{Instrumentos}

Ficha de Dados Sociodemográficos:

Utilizada para coletar informações como idade, sexo, religião, série escolar, trabalho, configuração familiar, repetência escolar, entre outros.

Escala Multidimensional de Satisfação de Vida para Adolescentes (EMSVA) (Segabinazi et al., 2010):

Escala composta por 52 itens que compõem sete dimensões da satisfação de vida de adolescentes: família, self, escola, self comparado, não violência, autoeficácia e amizade. As respostas são em escala Likert de cinco pontos, variando de 1 (nem um pouco) a 5 (muitíssimo). No estudo original (Segabinazi et al., 2010) foi encontrada uma adequada consistência interna da escala $(\alpha=0,93)$.

Escala de Afetos Positivos e Negativos para Adolescentes (EAPNA) (Segabinazi et al., 2012):

A escala constitui-se de 28 adjetivos descritores de estados afetivos subjetivos (14 positivos e 14 negativos), com opções de resposta em escala Likert de cinco pontos, variando de "nem um pouco" a "muitíssimo". No estudo de adaptação desse instrumento (Segabinazi et al., 2012), a solução bifatorial produziu o primeiro fator (AP) com eigenvalue de 8,1 que explicou $29 \%$ da variância total e o segundo fator (AN) com eigenvalue de 3,5 que explicou 12,4\% 
da variância total. A consistência interna medida pelo alfa de Cronbach foi de 0,88 para as ambas as subescalas.

Para verificar os níveis de bem-estar de forma global, foi construído um Índice de Bem-Estar (IBE), utilizando os escores padronizados da Escala Multidimensional de Satisfação de Vida para Adolescentes (EMSVA), somados aos escores padronizados da Escala de Afetos Positivos e Negativos para Adolescentes (EAPNA), tendo os itens de afetos negativos sido invertidos para o cálculo. Para construir o IBE, foi feita uma Análise dos Componentes Principais, pois esta permite criar um índice a partir das valências empíricas de seus componentes, ou seja, provenientes dos próprios dados (Field, 2009). O componente extraído (um único, pois a ideia é resumir em uma medida) explicou cerca de $75 \%$ da variância nos escores. A contribuição das variáveis, em ordem de importância foi de 0,91 para SV, 0,89 para AP e -0,79 para AN. Os componentes usados para criar o IBE são provenientes do modelo teórico proposto por Diener (1984), no qual entram a satisfação de vida, afetos positivos e afetos negativos. Para identificar os níveis de AP, AN, SV total e das dimensões de SV, foram somados os itens de AP e AN em separado. Para SV total, foram somados os itens da escala, invertendo-se aqueles que pesam negativamente para a satisfação de vida, a exemplo do item 11 "Brigo muito com meus amigos" e do item 20 "É difícil conseguir o que quero". Da mesma forma, para identificar os níveis de cada dimensão da escala, os itens das dimensões foram somados.

Destaca-se que a proposta de utilizar a EAPNA e a EMSVA em conjunto, as quais foram construídas para adolescentes, representa um avanço na avaliação do bem-estar subjetivo (Segabinazi et al., 2012). Estudo específico sobre a validação da construção do Índice de Bem-estar (IBE) está descrita em outro estudo (Silva, 2015) do qual deriva este artigo.

\section{Procedimentos e Considerações Éticas}

Os aspectos éticos que garantem a integridade dos participantes foram assegurados de acordo com legislações brasileiras, tendo como base a Resolução no 466 (Brasil, Conselho Nacional de Saúde, 2013). O estudo foi aprovado pelo Comitê de Ética em Pesquisa do Instituto de Psicologia da UFRGS, sob protocolo no 557.202. Foi realizado contato com a direção das escolas selecionadas por conveniência de diferentes regiões da cidade, as quais assinaram o Termo de Concordância da Instituição. Aos pais dos adolescentes participantes da pesquisa foi solicitada a assinatura do Termo de Consentimento Livre e Esclarecido (TCLE), assim como a assinatura de Termo de Assentimento por parte dos jovens. A aplicação dos questionários se deu forma coletiva nas escolas, com duração de aproximadamente 45 minutos. 


\section{RESULTADOS}

Neste estudo, a Escala Multidimensional de Satisfação de Vida apresentou índice de consistência interna de 0,87 (alfa de Cronbach). Esse mesmo índice foi de 0,89 e 0,88 para as Escalas de Afetos Negativos e Positivos respectivamente, demonstrando consistência satisfatória.

A Tabela 1 mostra os escores brutos e padronizados mínimo e máximo, observados em cada instrumento e no IBE.

\begin{tabular}{|c|c|c|c|c|}
\hline Medidas & $\begin{array}{c}\text { Satisfação de } \\
\text { Vida }\end{array}$ & $\begin{array}{c}\text { Afetos } \\
\text { Positivos }\end{array}$ & $\begin{array}{c}\text { Afetos } \\
\text { Negativos }\end{array}$ & $\begin{array}{c}\text { Índice Bem- } \\
\text { estar }\end{array}$ \\
\hline \multicolumn{5}{|l|}{ Escore Bruto } \\
\hline Mínimo & 100 & 16 & 14 & 69 \\
\hline Máximo & 256 & 70 & 63 & 311 \\
\hline Média & 199,60 & 50,91 & 24,77 & 225,72 \\
\hline$D P$ & 28,11 & 10,88 & 10,08 & 42,88 \\
\hline \multicolumn{5}{|c|}{ Escore Padronizado } \\
\hline Mínimo & $-1,52$ & $-2,42$ & $-0,86$ & $-3,37$ \\
\hline Máximo & 1,73 & 1,94 & 1,41 & 2,78 \\
\hline Média & $-0,00$ & $-0,01$ & 0,01 & $-0,00$ \\
\hline$D P$ & 0,56 & 0,76 & 0,44 & 1,00 \\
\hline
\end{tabular}

Quanto à religiosidade, foi observado que $69,7 \%$ dos adolescentes informaram ter alguma religião. A partir disso, foi usado o teste KolmogorovSmirnov, com auxílio do Programa SPSS, que indicou que as variáveis investigadas apresentaram distribuição normal $(p>0,05)$. Dessa forma, foram realizadas análises paramétricas (teste $t$ para amostras independentes), para verificar diferenças nos escores das variáveis de interesse por grupo, e o cálculo do tamanho do efeito, para verificar a magnitude das diferenças. Para tanto, foram considerados valores $d$ de Cohen para diferença de duas médias (pequeno $d=0,20$ a 0,30; médio $d=0,40$ a 0,70 e grande $d \geq 0,8$ ) (Cohen, 1988). Os dados são apresentados na Tabela 2. 
Tabela 2. Médias, Desvios-Padrão (entre parênteses), Testes $t$ e Tamanho do Efeito das Variáveis Investigadas por Grupo

\begin{tabular}{|c|c|c|c|c|c|}
\hline Variáveis & $\begin{array}{c}\text { Com religião } \\
(n=293)\end{array}$ & $\begin{array}{l}\text { Sem religião } \\
(n=127)\end{array}$ & $\mathrm{t}$ & $\mathrm{p}$ & $\begin{array}{l}\text { Tamanho } \\
\text { do ef eito }\end{array}$ \\
\hline $\begin{array}{l}\text { Satisfação de Vida } \\
\text { Total }\end{array}$ & $\begin{array}{r}201,76 \\
(26,55)\end{array}$ & $\begin{array}{r}194,33 \\
(30,96)\end{array}$ & 2,49 & 0,013 & 0,26 \\
\hline $\begin{array}{l}\text { Satisfação com a } \\
\text { Família }\end{array}$ & $\begin{array}{l}40,03 \\
(8,42)\end{array}$ & $\begin{array}{l}37,48 \\
(9,70)\end{array}$ & 2,57 & 0,011 & 0,29 \\
\hline $\begin{array}{l}\text { Satisfação com } \\
\text { Amizades }\end{array}$ & $\begin{array}{l}34,85 \\
(4,38)\end{array}$ & $\begin{array}{l}33,65 \\
(5,10)\end{array}$ & 2,30 & 0,022 & 0,26 \\
\hline $\begin{array}{l}\text { Índice de Bem- } \\
\text { estar Subjetivo }\end{array}$ & $\begin{array}{l}229,52 \\
(39,64)\end{array}$ & $\begin{array}{r}216,74 \\
(48,37)\end{array}$ & 2,62 & 0,010 & 0,30 \\
\hline Af etos Positivos & $\begin{array}{c}51,82 \\
(10,35)\end{array}$ & $\begin{array}{c}48,96 \\
(11,70)\end{array}$ & 2,49 & 0,013 & 0,26 \\
\hline Afetos Negativos & $\begin{array}{l}24,03 \\
(9,35)\end{array}$ & $\begin{array}{c}26,56 \\
(11,45)\end{array}$ & 2,19 & 0,029 & 0,25 \\
\hline
\end{tabular}

\section{DISCUSSÃo}

Primeiramente, é importante salientar que, apesar de muitos trabalhos na área do bem-estar, as definições conceituais e suas operacionalizações em termos de medida ainda são diversas (Albuquerque, \& Tróccoli, 2004; ScorsoliniComin, \& Santos, 2010). Além disso, a avaliação da religiosidade também tem se mostrado diversa entre os estudos, o que dificulta uma maior precisão do tema e dos dados encontrados. Segundo Pearce e Denton (2011), a forma ideal para avaliar religiosidade seria considerando o contexto, a conduta e a centralidade da prática religiosa na vida do indivíduo, elementos que não são contemplados nas pesquisas. Neste estudo, apenas foi considerado se o participante tinha alguma religião ou não, sendo que $30,3 \%$ deles indicaram não ter religião. Casas et al. (2015) também apontam que a inclusão de itens sobre satisfação com a religiosidade pode ser relevante ou não dependendo do contexto sociocultural dos participantes da pesquisa, especialmente quando ela é realizada em mais de um país.

A análise dos resultados indicou médias mais alta de satisfação de vida e bem-estar subjetivo entre quem relatou ter alguma religião, semelhante aos dados encontrados por outros pesquisadores (Abdel-Khalek, 2010; Silva \& Santos, 2013; Souza et al., 2012). Com efeito, quanto à religiosidade, cabe ressaltar a discussão de que essa poderia compor uma das dimensões da satisfação de vida, o que justifica considerá-la como um elemento importante na avaliação de BES de adolescentes (Sarriera et al., 2014). Dada sua relevância e associação com a satisfação de vida e bem-estar subjetivo, também encontrada neste estudo, aponta-se que tal evidência tem sido explicada pelo fato de que 
vivenciar a religiosidade proporciona suporte social, propósito e significado para a vida, fatores que incrementam o BES (Silva \& Santos, 2013).

A religião parece cumprir um papel positivo no desenvolvimento, visto que sua vivência é considerada fator protetivo para comportamentos de risco na adolescência (Van der Meer Sanchez, de Oliveira, \& Nappo, 2004). Há evidências de que os jovens experimentam mais bem-estar subjetivo quando se envolvem em atividades religiosas, pois nos locais dessas práticas encontram amigos, sentem-se seguros, são auxiliados quando estão passando por situações estressoras e sentem esperança (Eryilmaz, 2015). A prática de alguma religião também tem sido associada à adoção de comportamentos saudáveis, como atividades físicas, cuidar da saúde e não uso de álcool e outras drogas, devido à adesão de hábitos e estilo de vida relacionados com as religiões adotadas (Bezerra et al., 2009; Rew \& Wong, 2006). Esses aspectos de saúde, por sua vez, estão relacionados com bem-estar e satisfação com a vida (Park, 2004) e efeitos positivos na saúde mental dos adolescentes (Chatters et al., 2011).

O envolvimento com religião, em geral, é influenciado ou estimulado pela própria família do adolescente, o que tem sido evidenciado como uma forma de tornar os laços afetivos mais estreitos e minimizar os conflitos e desentendimentos intrafamiliares (Becker, Maestri, \& Bobato, 2015; Rabinovich, Costa, Lins, \& Franco, 2008). Pesquisa internacional longitudinal realizada com adolescentes portugueses (Marques, Lopez, \& Mitchell, 2013) indicou correlação entre religiosidade e fé e satisfação de vida total. Um estudo realizado com adolescentes chineses (Yuen, 2013) e outro estudo realizado com adolescentes americanos (Shannon et al., 2013) também encontraram essa correlação. Dessa forma, os resultados dessas pesquisas vão ao encontro dos achados relatados nesse trabalho, demonstrando que adolescentes que apresentam índices mais altos de religiosidade podem apresentar maiores níveis de satisfação de vida e bem-estar subjetivo.

Apesar da falta de consenso na forma de avaliação da religiosidade entre os adolescentes nas pesquisas, ter ou não religião pode servir como indicador da religiosidade e relaciona-se positivamente ao BES. Nesse sentido, são necessários mais estudos que avaliem religiosidade de forma mais precisa e sistemática, buscando compreender como se manifesta na adolescência, a exemplo da proposição de Pearce e Denton (2011). Dessa forma, deve-se analisar com cautela os resultados encontrados no presente estudo e em outras pesquisas, tendo em vista que as diferenças encontradas nas relações entre satisfação de vida, bem-estar e religiosidade podem estar relacionadas aos diferentes conceitos, instrumentos e metodologias utilizadas para sua avaliação.

Por outro lado, com relação à avaliação específica do bem-estar e seu componente de satisfação de vida, as pesquisas mostram evidências de que é possível mensurá-lo adequadamente e esse se mostra alto entre os adolescentes, apesar de os diferentes conceitos e instrumentos utilizados na área 
(Casas et al., 2015, Segabinazi et al., 2012). Os altos níveis de BES observados entre os adolescentes podem ser explicados pelo fato de que maioria dos jovens elenca a felicidade como muito importante em suas vidas (Diener, 2000), sendo algo almejado para lidar com as demandas da vida de forma mais positiva e adaptativa (De Neve et al., 2013). Desse modo, torna-se importante compreender com maior precisão o que os adolescentes levam em consideração ao avaliar sua satisfação de vida, com o intuito de promover o desenvolvimento positivo, uma vez que a presença de BES é recorrentemente associada à saúde e qualidade de vida (Passareli, \& Silva, 2007; Park, 2004). Ainda foi observado maior relato de afetos positivos entre os adolescentes que disseram ter religião. Esse resultado está de acordo com a ideia de que quanto maior a percepção de afetos positivos, melhor a avaliação positiva da satisfação de vida e BES (Diener \& Seligman, 2004).

Destaca-se, neste estudo, as médias de satisfação de vida, BES, afetos positivos, satisfação com a família e com as amizades mais altas entre os jovens que relataram possuir alguma religião. Esse dado vai ao encontro dos achados da literatura que indicam que as diferentes fontes de relacionamento interpessoal devem ser avaliadas em conjunto (Souza, \& Duarte, 2013). Souza e Duarte (2013) também sugerem que na avaliação da relação entre componentes do bem-estar e amizades devem ser incluídos os demais relacionamentos interpessoais, como os familiares e os amorosos. Os estudos têm apontado uma grande influência da família e do grupo de pares sobre os adolescentes, os quais podem funcionar como rede de proteção, favorecer o desenvolvimento saudável dos jovens e servir como fonte de apoio social, o que proporciona maior sentimento de bem-estar (Amparo et al., 2008; Segabinazi et al., 2012). A valorização das amizades pelos adolescentes, aspecto considerado característico dessa etapa do ciclo vital (Huebner et al., 2000; Sarriera et al., 2013; Serafini \& Bandeira, 2011), também incrementa o relato de maior satisfação de vida, tendo em vista que ela envolve componentes como companheirismo, ajuda, confiança e empatia (Souza \& Hutz, 2007).

Dessa forma, ao considerar a implicação da religiosidade, da satisfação com a família e com os amigos na satisfação de vida total e no BES em adolescentes, apontam-se evidências de que estas dimensões podem contribuir para a promoção de um elemento positivo, especificamente para esta faixa etária. Por sua vez, a promoção do BES vai ao encontro da proposta de valorizar aspectos positivos e de proteção dos adolescentes, o que corresponde à retomada do eixo principal da psicologia positiva (Diener, 2000; Diener, \& Scollon, 2014). 


\section{CONSIDERAÇÕES FINAIS}

Este estudo teve por objetivo discutir as relações entre satisfação de vida, satisfação com a família e com as amizades e religiosidade, em amostra de adolescentes de escolas públicas de Porto Alegre. As análises realizadas revelaram médias altas de satisfação de vida e bem-estar subjetivo entre adolescentes que disseram possuir alguma religião, o que indica que este fator pode atuar de forma positiva na avaliação que o sujeito faz da própria vida. Da mesma forma, aqueles que disseram ter religião tiveram médias significativamente mais altas na satisfação com as amizades e com a família, indicando a relevância das variáveis religiosidade e satisfação com relacionamentos na avaliação da satisfação de vida e, por consequência, do BES. Destaca-se que altos níveis de bem-estar na adolescência facilitam a passagem do jovem em meio a um período de intensas transformações. Neste estudo a satisfação de vida, os afetos positivos, satisfação com a família, com as amizades e a presença de religiosidade foram identificados como fatores importantes do bem-estar subjetivo para os adolescentes da amostra.

Neste sentido, incrementa-se a importância das evidências científicas sobre os fatores que compõem a satisfação de vida e o BES de adolescentes, bem como de aspectos que podem ser trabalhados para promovê-lo. Para tanto, é necessária clareza na definição conceitual do bem-estar, na identificação de seus componentes e na forma de avaliá-lo, para uma maior confiabilidade dos dados (Scorsolini-Comin \& Santos, 2010). Além disso, são fundamentais estudos que forneçam evidências de validade de construto, concorrente e de fidedignidade, como o de Albuquerque e Tróccoli (2004), assim como pesquisas com amostras de adolescentes como a de Segabinazi et al. $(2012,2010)$.

Destaca-se o cuidado ao selecionar a forma de avaliação de BES, que deve considerar o conceito subjacente. O modelo de Diener (1984) tem como pressuposto que a medida é realizada por autorrelato através de diferentes escalas, as quais têm mostrado propriedades psicométricas satisfatórias (Albuquerque \& Tróccoli, 2004; Giacomoni \& Hutz, 2008; Segabinazi et al., $2010 ; 2012)$. No entanto, questiona-se se essa avaliação poderia incluir algum outro tipo de medida que não envolva apenas a percepção do próprio indivíduo, uma vez que a vivência dos afetos se caracteriza por ser mais imediata e subjetiva em resposta a fatores do ambiente, o que torna a avaliação dos afetos e da satisfação de vida mais lábil (Diener, Smith, \& Fujita, 1995). Dessa forma, conforme discutem Scorsolini-Comin e Santos (2010), seria importante poder avançar nos estudos de modo a obter dados empíricos que delimitem com mais profundidade o conceito e considerem outras formas pelas quais os adolescentes possam apreender e relatar a percepção do BES.

Além disso, enfatiza-se a necessidade de que sejam desenvolvidos instrumentos capazes de avaliar com mais propriedade a religiosidade do 
indivíduo, assim como seu nível de envolvimento religioso, a fim de investigar a interação dessa variável com o bem-estar na adolescência. Destaca-se ainda que no presente estudo apenas foi avaliado se o adolescente tinha ou não alguma religião, e não especificamente se era ateu, o que também pode gerar diferença e ter influenciado a avaliação da religiosidade pelos adolescentes e afetar a avaliação do BES. De fato, na amostra deste trabalho, os jovens que disseram não ter nenhuma religião apresentaram menores níveis de satisfação de vida. Dessa forma, também poderia ser mais bem investigada a necessidade de incluir a dimensão religiosidade na avaliação do BES, tendo em vista a sua associação demonstrada nesse e em outros trabalhos.

Contudo, no que se refere às limitações do presente estudo, destaca-se que, embora tenham sido encontradas diferenças significativas entre os grupos, os tamanhos de efeito verificados são pequenos e considerados baixos (Cohen, 1988). Além disso, deve-se observar que outras variáveis que não foram controladas, como sexo e idade, podem ser fatores intervenientes nos processos investigados. Sendo assim, os resultados descritos aqui devem ser interpretados com cautela ao inferir relações de causalidade entre os fenômenos estudados, uma vez que as medidas utilizadas não se prestam a esse tipo de conclusão.

Como sugestão para futuros estudos, indica-se a continuidade do uso das escalas para avaliação da satisfação de vida e do BES utilizadas na presente pesquisa, a fim de verificar suas propriedades psicométricas e estabilidade da medida do construto. A validade do IBES deve ser mais bem investigada em trabalhos de validação do IBES, bem como sua utilização em estudos correlacionais. Paralelamente, sugere-se delineamentos que envolvam algum tipo de medida diferente das de autorrelato para poder verificar se entrevistas ou outros métodos qualitativos seriam mais sensíveis a variações pessoais na avaliação do BES e se tais dados qualitativos estariam associados às medidas quantitativas fornecidas pelos instrumentos padronizados.

Destaca-se que os resultados aqui encontrados não podem ser generalizados e que se tornam importantes novos estudos com amostras diversificadas, como em diferentes regiões como cidades de capital e de interior de estado, a fim de verificar possíveis diferenças culturais no contexto urbano e rural. Ademais, a ampliação e diversificação das amostras em estudos também se justificam devido a características específicas dos contextos de inserção de jovens de escolas públicas e privadas (Fleith, \& Alencar, 2006; Jatobá, \& Bastos, 2007).

\section{REFERÊNCIAS}

Abdel-Khalek, A. M. (2010). Quality of life, subjective well-being, and religiosity in Muslim college students. Quality of Life Research, 19(8), 1133-1143. doi: 10.1007/s11136-010-9676-7. 
Albuquerque, A. S., \& Tróccoli, B. T. (2004). Desenvolvimento de uma escala de bem-estar subjetivo. Psicologia: Teoria e Pesquisa, 20(2), 153-164. doi: $10.1590 /$ S0102-37722004000200008

Amparo, D. M., Galvão, A. C. T., Alves, P. B., Brasil, K. T., \& Koller, S. H. (2008). Adolescentes e jovens em situação de risco psicossocial: Redes de apoio social e fatores pessoais de proteção. Estudos de Psicologia, 13(2), 165174. doi:10.1590/S1413-294X2008000200009

Becker A. P. S., Maestri, T. P., \& Bobato, S. T. (2015). Impacto da religiosidade na relação entre pais e filhos adolescentes. Arquivos Brasileiros de Psicologia, 67(1), 84-98.

Bezerra, J., Barros, M. V. G., Tenório, M. C. M., Tassitano, R. M., Barros, S. S. H., \& Hallal, P. C. (2009). Religiosidade, consumo de bebidas alcoólicas e tabagismo em adolescentes. Revista Panamericana de Salud Pública, 26(5), 440-446. doi:10.1590/S1020-49892009001100009

Brasil. Conselho Nacional de Saúde (2013). Diretrizes e normas para pesquisa envolvendo seres humanos. RESOLUÇÃO No 466, de 12 de dezembro de 2012. Brasília, DF: Diário Oficial da União; Poder Executivo, publicado em 13 jun. 2013. Seção I, p.59-62.

Casas, F., Alfaro, J., Sarriera, J. C, Bedin, L., Grigoras, B., Bălţătescu, S.,... Sirlopú, D. (2015). El bienestar subjetivo en la infancia: Estudio de la comparabilidad de 3 escalas psicométricas en 4 países de habla latina. Psicoperspectivas, 14(1), 6-18. doi:10.5027/psicoperspectivas-Vol14Issue1-fulltext-522

Casas, F., Sarriera, J. C., Alfaro, J., González, M., Bedin, L., Abs, D., ... Valdenegro, B. (2015). Reconsidering life domains that contribute to subjective well-being among adolescents with data from three countries. Journal of Happiness Studies, 16(2), 491-513. doi:10 10.1007/s902-0149520-9

Chatters, L. M., Mattis, J. S., Woodward, A. T., Taylor, R. J., Neighbors, H. W., \& Grayman, N. A. (2011). Use of ministers for a serious personal problem among African Americans: Findings from the national survey of American life (NSAL). American Journal of Orthopsychiatry, 81(1), 118-127. doi: $10.1111 / \mathrm{j} .1939-0025.2010 .01079 . x$

Cheng, S. T., Li, K. K., Leung, E. M. F. \& Chan, A. C. M. (2011). Social exchanges and subjective well-being: Do sources of positive and negative exchanges matter? Journals of Gerontology Series B-Psychological Sciences and Social Sciences, 66(6), 708-718. doi:10.1093/geronb/gbr061.

Coenders, G., Casas, F., Figuer, C., \& González, M. (2005). Relationships between parents' and children's salient values for future and children's overall satisfaction. A comparison across countries. Social Indicators Research, 73, 141-177. doi:10.1007/s11205-004-3233 
Cohen, J. (1988). Statistical power for behavioral sciences. New York, NY: Academic Press.

De Neve, J. E., Diener, E. Tay, L., \& Xuereb, C. (2013). The objective benefits of subjective well-being. In J. Helliwell, R. Layard, \& J. Sachs (Eds.), World Happiness Report 2013 (pp. 54-79). New York, NY: UN Sustainable Development Solutions Network.

Diener, E. (1984). Subjective well-being. Psychological Bulletin, 95, 542-575.

Diener, E. (2000). Subjective well-being: The science of happiness and a proposal for a national index. American Psychologist, 55(1), 34-43. doi: $10.1037 / 0003-066 \times .55 .1 .34$

Diener, E., \& Scollon, C. (2014). The what, why, when, and how of teaching the science of subjective well-being. Teaching of Psychology, 41, 175-183. doi: $10.1177 / 0098628314530346$

Diener, E., \& Seligman, M. E. P. (2004). Beyond money: Toward an economy of well-being. Psychological Science in the Public Interest, 5(1), 1-31.

Diener, E., Smith, H., \& Fujita, F. (1995). The personality structure of affect. Journal of Personality \& Social Psychology, 69, 130-141.

Eryilmaz, A. (2015). Investigation of the relations between religious activities and subjective well-being of high-school students. Educational ScienceTheory \& Practice, 15(2), 433-444. doi:10.12738/estp.2015.2.2327

Field, A. (2009). Análise de fatores exploratória. In Descobrindo a estatística usando o SPSS (pp. 553-604). Porto Alegre: Artmed.

Fleith, D. S., \& Alencar, E. M. L. S. (2006). Percepção de alunos do ensino fundamental quanto ao clima de sala de aula para criatividade. Psicologia em Estudo, 11(3), 513-521. doi:10.1590/S1413-73722006000300007

Giacomoni, C. H., \& Hutz, C. S. (2008). Escala multidimensional de satisfação de vida para crianças: Estudos de construção e validação. Estudos de Psicologia (Campinas), 25(1), 23-35. doi:10.1590/S0103166X2008000100003

Huebner, E. S., Drane, W., \& Valois, R. F. (2000). Levels and demographic correlates of adolescent life satisfaction reports. School Psychology International, 21, 281-292. doi:10.1177/0143034300213005

Jatobá, J. D. V. N., Bastos, O. (2007). Depressão e ansiedade em adolescentes de escolas públicas e privadas. Jornal Brasileiro de Psiquiatria, 56(3), 171179. doi: $10.1590 /$ S0047-20852007000300003

Marques, S. C., Lopez, S. J., \& Mitchell, J. (2013). The role of hope, spirituality and religious practice in adolescents' life satisfaction: Longitudinal findings. Journal of Happiness Studies, 14(1), 251-261. doi:10.1007/s10902-0129329-3

Park, N. (2004). The role of subjective well-being in positive youth development. The Annals of the American Academy of Political and Social Science, 591(1), 25-39. doi:10.1177/0002716203260078 
Passareli, P. M., \& Silva, J. A. (2007). Psicologia positiva e o estudo do bem-estar subjetivo. Estudos de Psicologia (Campinas), 24(4), 513-517. doi: $10.1590 / S 0103-166 \times 2007000400010$

Pearce, L., \& Denton, M. L. (2011). A faith of their own: Stability and change in the religiosity/spirituality and adolescent health attitudes and behaviors. Journal of Adolescent Health, 38, 433-442. Recuperado de: https://books.google.com.br/books

Rabinovich, E. P., Costa, L. A. F., Lins, A., \& Franco, S. (2008). Famílias evangélicas baianas e o processo de nomeação. Psicologia e Sociedade, 20(3), 417-424. doi:10.1590/S0102-71822008000300012

Reina, M. C., Oliva, A., \& Parra, A. (2010). Percepciones de autoevaluación: Autoestima, autoeficacia y satisfacción vital en la adolescencia. Psychology, Society, \& Education, 2, 55-69.

Rew, L., \& Wong, Y. J. (2006). A systematic review of associations among religiosity/spirituality and adolescent health attitudes and behaviors. Journal of Adolescent Health, 38, 433-442.

Ryan, R. M. \& Deci, E. L. (2001). On happiness and human potentials: A review of research on hedonic and eudaimonic well-being. Annual Review of Psychology, 52, 141-166. doi:10.1146/annurev.psych.52.1.141

Sarriera, J. C., Casas, F., Alfaro, J., Bedin, L., Strelhow, M. R. W., Abs, D., Valdenegro, B., García, C., \& Oyarzún, D. (2014). Psychometric properties of the personal wellbeing index in Brazilian and Chilean adolescents including spirituality and religion. Psicologia: Reflexão e Crítica, 27(4), 710719. doi: $10.1590 / 1678-7153.201427411$

Sarriera, J. C., Paradiso, A. C., Abs, D., Soares, D. H., Silva, C. L. E., \& Fiuza, P. J. (2013). O bem-estar pessoal dos adolescentes através do seu tempo livre. Estudos de Psicologia, 18, 285-295. doi:10.1590/S1413294X2013000200014

Sarriera, J. C., Saforcada, E., Tonon, G., Vega, L. R. L., Mozobancyk, S., \& Bedin, L. (2012). Bienestar subjetivo de los adolescentes: Un estudio comparativo entre Argentina y Brasil. Psychosocial Intervention, 21, 273-280. doi: 10.5093/in2012a24

Scorsolini-Comin, F., \& Santos, M. A. (2010). Avaliação do bem-estar subjetivo (BES): Aspectos conceituais e metodológicos. Interamerican Journal of Psychology, 44(3), 442-448.

Segabinazi, J. D., Giacomoni, C. H., Dias, A. C. G, Teixeira, M. A. P., \& Moraes, D. A. O. (2010). Desenvolvimento e validação preliminar de uma escala multidimensional de satisfação de vida para adolescentes. Psicologia: Teoria e Pesquisa, 26(4), 653-659. doi:10.1590/S0102-37722010000400009 
Segabinazi, J. D., Zortea, M., Zanon, C., Bandeira, D. R., Giacomoni, C. H., \& Hutz, C. S. (2012). Escala de afetos positivos e negativos para adolescentes: Adaptação, normatização e evidências de validade. Avaliação Psicológica, 11(1), 1-12.

Serafini, A. J., \& Bandeira, D. N. (2011). A influência da rede de relações, do coping e do neuroticismo na satisfação de jovens estudantes. Estudos de Psicologia, 28(1), 15-25. doi:10.1590/S0103-166X2011000100002

Shannon, D. K., Oakes, K. E., Scheers, N. J., Richardson, F. J., \& Stills, A. B. (2013). Religious beliefs as moderator of exposure to violence in African American adolescents. Psychology of Religion and Spirituality, 5(3), 172. doi: $10.1037 / \mathrm{a} 0030879$

Silva, D. G. (2015). Relações entre exposição à violência e bem-estar subjetivo em adolescentes. Dissertação de Mestrado, Programa de Pós-Graduação em Psicologia, Universidade Federal do Rio Grande do Sul, Porto Alegre, RS, Brasil.

Silva, J. A. \& Santos, R. C. (2013). Bem-estar subjetivo, educação, inteligência e religião. Temas em Psicologia, 21(2), 519-523. doi:10.9788/TP2013.2-17.

Souza, L. D. D. M., Maragalhoni, T. D. C., Quincoses, M. T., Jansen, K., Cruzeiro, A. L. S., ..., \& Pinheiro, R. T. (2012). Bem-estar psicológico de jovens de 18 a 24 anos: Fatores associados. Cadernos de Saúde Pública, 28(6), 11671174. doi:10.1590/S0102-311X2012000600015

Souza, L. K., \& Duarte, M. G. (2013). Amizade e bem-estar subjetivo. Psicologia: Teoria e Pesquisa, 29(4), 429-436. doi:10.1590/S010237722013000400009

Souza, L. K. de, \& Hutz, C. S. (2007). A qualidade da amizade: Adaptação e validação dos Questionários McGill. Aletheia, 25,82-96. doi:10.1590/S010237722013000400009

Tomé G., Camacho I., Matos M. G., \& Simões C. (2015). Influence of family and friends in wellbeing and risk behavior: Explanatory model. Psicologia, Saúde \& Doenças, 16(1), 23-34. doi:10.15309/15psd160104.

Tomé, G., Camacho, I., Matos, M. D., \& Diniz, J. A. (2011). A influência da comunicação com a família e grupo de pares no bem-estar e nos comportamentos de risco nos adolescentes portugueses. Psicologia: Reflexão e Crítica, 24(4), 747-756. doi:10.1590/S010279722011000400015

Van der Meer Sanchez, Z., de Oliveira, L. G., \& Nappo, S. A. (2004). Fatores protetores de adolescentes contra o uso de drogas com ênfase na religiosidade. Ciência \& Saúde Coletiva, 9(1), 43-55. doi:10.1590/S141381232004000100005

Watson, D., Clark, L. A., \& Tellegen, A. (1988). Development and validation of brief measures of positive and negative affect: The PANAS scales. Journal of Personality and Social Psychology, 54(6), 1063-1070. doi:10.2307/2137092 
Wills, E. (2009). Spirituality and subjective well-being: Evidences for a new domain in the personal well-being index. Journal of Happiness Studies, 10(1), 49-69. doi:10.1007/S10902-007-9061-6

Yuen, C. Y. (2013). Ethnicity, level of study, gender, religious affiliation and life satisfaction of adolescents from diverse cultures in Hong Kong. Journal of Youth Studies, 16(6), 776-791. doi:10.1080/13676261.2012.756973

\section{Sobre os autores}

Doralúcia Gil da Silva é psicóloga pela Universidade Federal do Rio Grande do Sul, mestre e doutoranda em Psicologia pela Universidade Federal do Rio Grande do Sul. Trabalha como psicóloga hospitalar da linha materno infantil do Hospital Escola da Universidade Federal de Pelotas. doralu.gil@gmail.com Jaqueline Portella Giordani é psicóloga pela Universidade Federal do Rio Grande do Sul, mestre e doutoranda em Psicologia pela Universidade Federal do Rio Grande do Sul. Trabalha como psicóloga no Colégio de Aplicação da Universidade Federal do Rio Grande do Sul. jaquelinegiordani@gmail.com

Débora Dalbosco Dell'Aglio é psicóloga pela Pontifícia Universidade Católica do Rio Grande do Sul, mestre e doutora em Psicologia pela Universidade Federal do Rio Grande do Sul. Trabalha como professora do Programa de Pós-Graduação em Psicologia da Universidade Federal do Rio Grande do Sul. dddellaglio@gmail.com

Recebido em: 24/03/2016 $1^{\text {a }}$ revisão em: 02/05/2016 $2^{a}$ revisão em: 30/09/2016 Aceito em: 03/11/2016 